\title{
Evaluation of pharmacokinetics of warfarin from validated pharmacokinetic-pharmacodynamic model
}

\author{
Kannan Sridharan ${ }^{1 *}$, Rashed Al Banna ${ }^{2}$, Aysha Husain ${ }^{2,3}$ \\ ${ }^{1}$ Department of Pharmacology \& Therapeutics, College of Medicine \& Medical Sciences, Arabian Gulf University, \\ Manama, Kingdom of Bahrain. \\ ${ }^{2}$ Department of Cardiology, Salmaniya Medical Hospital, Ministry of Health, Manama, Kingdom of Bahrain. \\ ${ }^{3}$ RCSI-MUB, Manama, Kingdom of Bahrain Full Affiliation, Address
}

*Corresponding Author: E-mail: skannandr@gmail.com; ORCID-ID: 0000-0003-3811-6503

Received: September 21, 2020; Revised: January 06, 2021; Published online: January 18, 2021

\begin{abstract}
Background: Pharmacokinetics of warfarin has not been described in our population. We derived the pharmacokinetic parameters from a validated pharmacokinetic-pharmacodynamic model. Methods: Patients receiving warfarin for at least 6 months were recruited and their demographic characteristics, prothrombin time international normalized ratio (PT-INR), warfarin doses and concomitant drugs were collected. Using a validated pharmacokinetic-pharmacodynamic model, we predicted maximum plasma concentration $\left(C_{\max }\right)$, total clearance $\left(C_{\mathrm{L}}\right)$, volume of distribution $\left(V_{\mathrm{d}}\right)$ and elimination rate $(k)$. Warfarin sensitive index (WSI) and warfarin composite measures (WCM) were estimated from the dose and INR values. Liver weight was predicted using validated formula. Results: Two-hundred and twenty patients were recruited. The following were the predicted pharmacokinetic parameters: $C_{\max }(\mathrm{mg} / \mathrm{L})$ was $5.8(0.4) ; k$ (L/day) was 1 (0.1); $C L$ (L/day) was 2.1 (0.2); and $V_{d}(L)$ was $7.6(0.2)$. Patients with $C_{\max }$ and elimination rate outside the mean+1.96 SD had significantly lower WSI and higher WCM. Significant correlations were observed between $C_{\max }$ with $\mathrm{CL}, V_{\mathrm{d}}$, and $k$ of warfarin. Significant correlations were also observed between $\mathrm{CL}$ and $V_{d}$ of warfarin with liver weight of the study participants. Conclusion: We predicted pharmacokinetic parameters of warfarin from the validated pharmacokinetic-pharmacodynamic model in our population. More studies are needed exploring the relationship between various pharmacodynamic indices of warfarin and pharmacokinetic parameters of warfarin.
\end{abstract}

C2021 by the authors. This article is an open-access article distributed under the terms and conditions of the Creative Commons Attribution license (http://creativecommons.org/licenses/by/4.0/).

\section{Keywords}

Pharmacokinetics; Warfarin; Anticoagulant

\section{Introduction}

Warfarin, an oral anticoagulant, exhibits narrow therapeutic window and poses clinical challenges in maintaining appropriate therapeutic effect. Warfarin is metabolized in liver and its anticoagulant effect is considered optimum when the prothrombin time international normalized ratio (PT-INR) is to be maintained between 2.5 and 3.5 for those undergoing heart valve replacements and between 2 and 3 for others [1]. Warfarin is completely absorbed with time to maximum concentration of 2-6 hours; volume of distribution $\left(V_{d}\right)$ of $10 \mathrm{~L}$; clearance $(\mathrm{CL})$ of 0.2 
L/hour; and elimination half-life of 35 hours [2]. Warfarin is a racemic mixture of $S$ - and $R$-warfarin posing challenges in measurements [3]. Pharmacokinetic-pharmacodynamic (PK-PD) relationship of warfarin has been well elucidated and therapeutic drug monitoring is carried out by PT-INR measurement [4]. Warfarin pharmacokinetics has not been described in our population. Hence, we carried out the present study to predict the pharmacokinetic parameters of warfarin from the pharmacodynamic variables using a validated PD-PD model.

\section{Experimental}

\section{Study ethics and design}

The study was retrospective carried out as a part of warfarin pharmacogenomics study after obtaining approval from institutional ethics committee and consent from study participants. The present study complies with the latest World Medical Association Declaration of Helsinki guidelines.

\section{Study procedure}

Participants receiving warfarin for at least 6 months were recruited in the present study. Their demographics, PT-INR values, dose and frequency of warfarin, and concomitant drugs were obtained. We assessed $\mathrm{CHA}_{2} \mathrm{DS}_{2}$-VASc, HASBLED and SAMe-TT2R2 scores for the study participants [5,6]. Drugs/drug classes such as statins, proton pump inhibitors, carbamazepine and amiodarone were categorized as drugs with potential interaction with warfarin [7]. We followed National Institute for Health and Care Excellence (NICE) guidelines for classifying anticoagulation control into good (TTR $\geq 65 \%$ ) and poor (TTR < $65 \%$ ) [8]. We estimated Warfarin sensitive index (WSI) based on the ratio of PT-INR upon the last dose of warfarin [9]. We assessed the difference in PT-INR between the two consequent visits that was squared and divided by the time interval in days. This cross product with the average of PT-INR on the visits followed by square root of this product provided the INR variability. We log-transformed it to base 10 to obtain log-INR variability [10]. Standardized Z scores were obtained for TTR and log-INR variability and warfarin composite measure (WCM) was estimated [11]. Liver weight was estimated by using the validated formula: liver weight $(\mathrm{g})=218+$ body weight $(\mathrm{kg}) * 12.3+$ gender $* 51$ (Male-1; Female-0) [12].

\section{Predicted pharmacokinetic parameters}

We used JPKD software ${ }^{\odot}$ for estimating pharmacokinetic parameters of warfarin from the validated warfarin PD-PD inbuilt model [13]. The following equations were input in the model:

$$
\begin{aligned}
& C_{\mathrm{s}}=\left(\left(1 /\left(-\left(\mathrm{m}^{*} \mathrm{Cl} / V\right) /\left(k^{\wedge} 2\right) *\left(1-\left(k^{*} \mathrm{Tau} / 24\right) /\left(1-\exp \left(-k^{*} \mathrm{Tau} / 24\right)\right)\right)-(m / k) * \ln \left((\text { Dose } / V) /\left(C_{\max }{ }^{*}(1-\exp (-\right.\right.\right.\right.\right. \\
& \left.\left.\left.\left.\left.\left.(\mathrm{Cl} / \mathrm{V})^{*}(\mathrm{Tau} / 24)\right)\right)\right)\right)+3.36\right) / 4.368\right)^{\wedge}(1 / 0.383) \\
& \text { INR }=\left(\left(1 /\left(-\left(m^{*} \mathrm{Cl} / V\right) /\left(k^{\wedge} 2\right) *\left(1-\left(k^{*} \operatorname{Tau} / 24\right) /\left(1-\exp \left(-k^{*} \mathrm{Tau} / 24\right)\right)\right)-(m / k) * \ln \left((\text { Dose } / V) /\left(C_{\max } *(1-\exp (-\right.\right.\right.\right.\right. \\
& (\mathrm{Cl} / \mathrm{V}) *(\mathrm{Tau} / 24))))))+3.36) / 4.368)^{\wedge}(1 / 0.383) \\
& \text { Dose }=\exp \left(\left(1+\left(\left(m^{*} \mathrm{Cl} / V\right) / k^{\wedge} 2\right) *\left(1-\left(k^{*} \mathrm{Tau} / 24\right) /\left(1-\exp \left(-k^{*} \mathrm{Tau} / 24\right)\right)\right) *\left(4.368^{*} \operatorname{INR}^{\wedge}(0.383)-3.36\right)\right) /((-\right. \\
& \left.\left.m / k) *\left(4.368 * \operatorname{INR}^{\wedge}(0.383)-3.36\right)\right)\right) * C_{\max } *(1-\exp ((-\mathrm{Cl} / V) * \mathrm{Tau} / 24)) * V
\end{aligned}
$$

\section{Statistical analysis}

We represented the demographic details using descriptive statistics. Chi-square test for association was used for assessing the categorical variables. Numerical variables following the assessment of their distributions were tested using Mann-Whitney $U$ test. Correlations between the variables were tested using Pearson correlation tests. A p-value of $\leq 0.05$ was considered significant. SPSS version 26 (IBM Corp. 
Released 2019. IBM SPSS Statistics for Windows, Version 26.0. Armonk, NY: IBM Corp.) was used for statistical analysis. Normal distributions were created for each of the key pharmacokinetic parameters and characteristics were compared between those with values within mean \pm 1.96 SD and those outside this range.

\section{Results}

\section{Demographics}

Two-hundred and twenty patients were enrolled in this study. Their demographic details are listed in Table 1. The concomitant diagnoses in the study participants were as follows: systemic hypertension $(n=130)$, diabetes mellitus $(n=98)$, atrial fibrillation $(n=85)$, ischemic heart disease $(n=55)$, thyroid disorders $(n=24)$, congestive cardiac failure $(n=9)$ and bronchial asthma $(n=15)$.

Table 1. Demographic details of the study participants $(\mathrm{N}=220)$

\begin{tabular}{|c|c|}
\hline Variables & Values \\
\hline Age (years) & $66.6(13.4)$ \\
\hline Male : Female & $1: 1$ \\
\hline Body weight $(\mathrm{kg})^{\$}$ & $75.2(18.1)$ \\
\hline Predicted liver weight $(\mathrm{g})^{\$}$ & $1148(265)$ \\
\hline $\mathrm{CHA}_{2} \mathrm{DS}_{2}$-VASc score & $3.7(1.5)$ \\
\hline HASBLED score $\$$ & $2.3(1.1)$ \\
\hline SAMe- $\mathrm{TT}_{2} \mathrm{R}_{2}$ score ${ }^{\mathrm{S}}$ & $1.4(0.6)$ \\
\hline Duration of warfarin (days) ${ }^{\$}$ & $1027(572.2)$ \\
\hline Warfarin sensitive index $\$$ & $0.7(0.4)$ \\
\hline Log INR variability $\$$ & $-0.84(0.2)$ \\
\hline Warfarin composite measure score ${ }^{\$}$ & $-1.3(1.2)$ \\
\hline $\begin{array}{l}\text { Number of patients with drugs with } \\
\text { potential interaction [n (\%)] }\end{array}$ & $136(61.8)$ \\
\hline
\end{tabular}

\section{Pharmacokinetic parameters}

Mean (SD) $C_{\max }(\mathrm{mg} / \mathrm{L})$ amongst the study participants was $5.8(0.4)$; elimination rate (L/day) was 1 (0.1); $\mathrm{CL}$ (L/day) was 2.1 (0.2); and $V_{d}$ (L) was 7.6 (0.2). Distributions of the pharmacokinetic parameters are depicted in Figure 1. Nineteen patients had their $C_{\max }$ outside the mean $\pm 1.96 \mathrm{SD}$ and 14 had the same for elimination rate. Such patients were observed with significantly lower WSI and

greater WCM (Table 2). For $\mathrm{CL}$, two had their values lower than mean-1.96 SD; and for $V_{\mathrm{d}}$, two had lower than mean-2 SD, and four more than mean+2 SD. Due to the number constraints, we could not analyze the differences in any of the variables for $C L$ and $V_{d}$ of warfarin.

No significant differences were observed between the predicted pharmacokinetic parameters across the age groups (Figure 2). Similar values were obtained for males $\left[C_{\max }(\mathrm{mg} / \mathrm{L}-5.8(0.4) ; k\right.$ (per day) $-1(0.1) ; \mathrm{CL}$ (L/day) - $2.1(0.2) ;$ and $\left.V_{d}(\mathrm{~L})-7.6(0.2)\right]$ and females $\left[C_{\max }(\mathrm{mg} / \mathrm{L}-5.8(0.4) ; k\right.$ (per day) $-1(0.05) ; \mathrm{CL}$ (L/day) - $2.1(0.2)$; and $V_{d}(L)-7.7(0.2)$ ]. Significant correlations were observed between $C_{\max }$ and $C L(r=-$ $0.4 ; p=0.0001), V_{d}(r=0.28 ; p=0.004)$ and $k(r=0.8 ; p=0.0001)$ (Figure 3).

\section{Association between liver weights and predicted pharmacokinetic parameters}

Scatterplot revealed significant correlations between warfarin $C L(r=0.2 ; p=0.04)$ and $V_{d}(r=-0.2 ; p=0.03)$ with weight of the liver (Figure 3). 
(A)

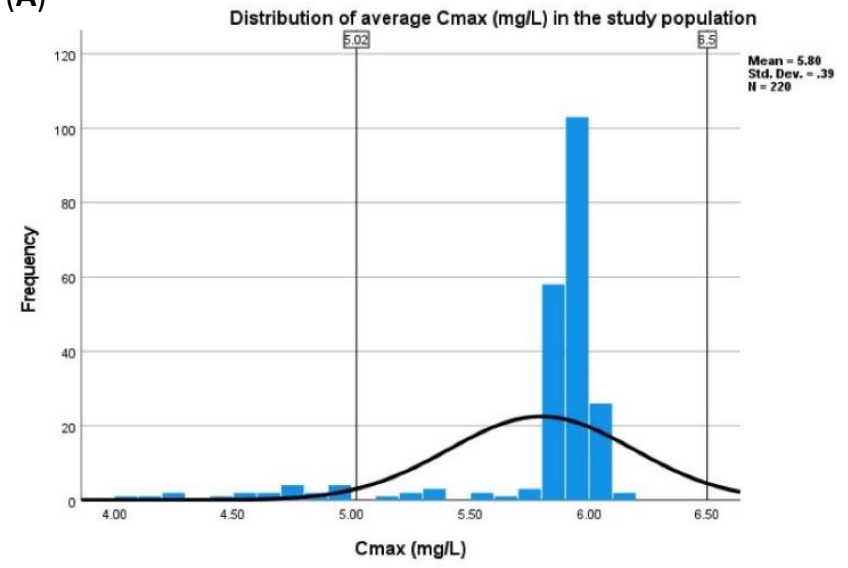

(C)

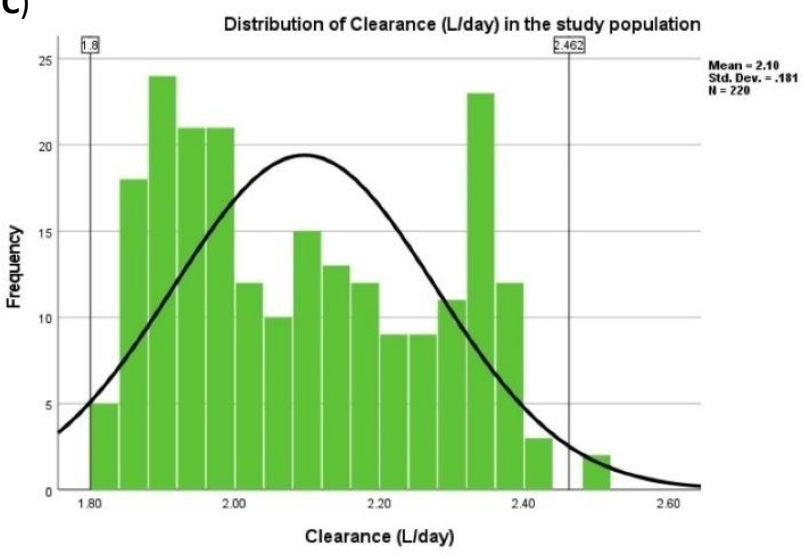

(B)

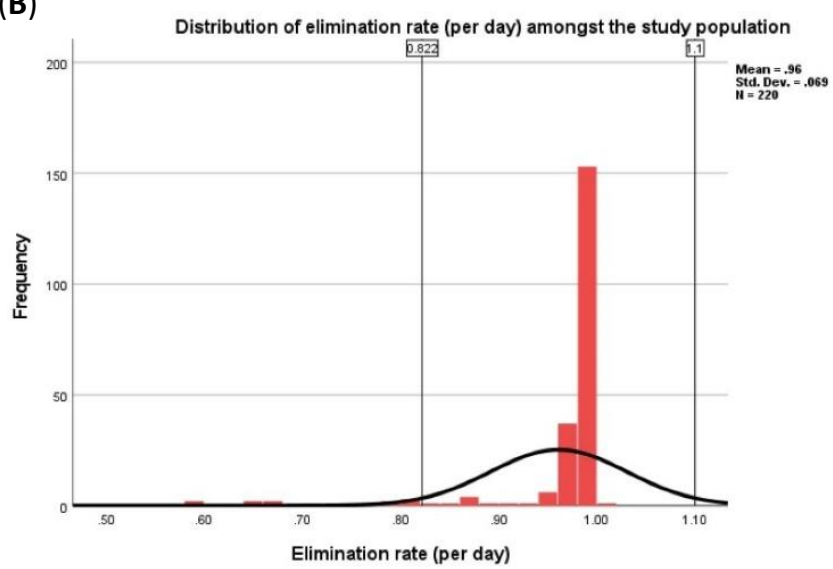

(D)

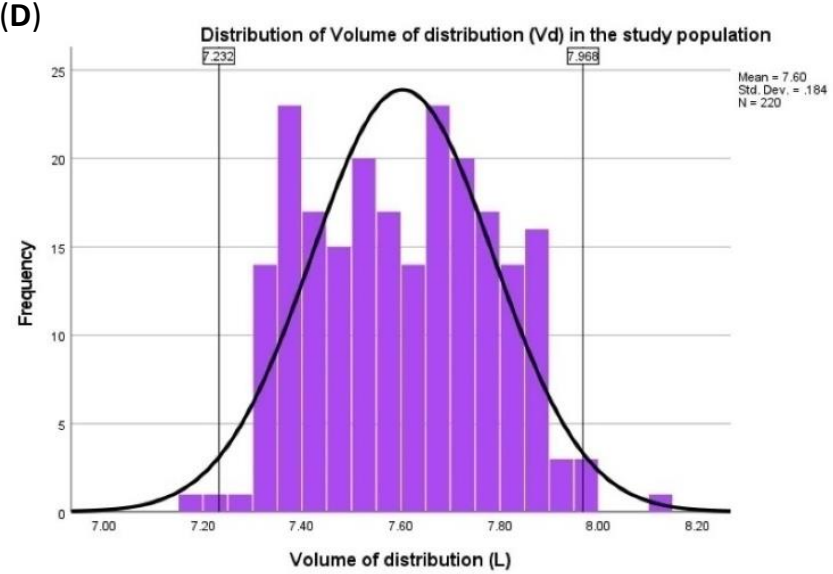

Figure 1. Distribution of pharmacokinetic parameters in the study population. (A) Distribution of $C_{\max }(\mathrm{mg} / \mathrm{L})$; (B) elimination rate (per day); (C) clearance (L/day); (D) volume of distribution (L) in the study population

Table 2. Comparison of characteristics between those within and outside $95 \%$ of normal distribution curves in the study population

\begin{tabular}{|c|c|c|c|c|c|c|}
\hline \multirow[b]{2}{*}{ Variables } & \multicolumn{3}{|c|}{$C_{\max }(\mathrm{mg} / \mathrm{L})$} & \multicolumn{3}{|c|}{ Elimination rate (per day) } \\
\hline & $\begin{array}{c}\text { Within } 95 \% \text { of } \\
\text { ND }(n=201)\end{array}$ & $\begin{array}{l}\text { Outside } 95 \% \\
\text { of ND }(n=19)\end{array}$ & P-values & $\begin{array}{c}\text { Within } 95 \% \text { of } \\
\text { ND }(n=206)\end{array}$ & $\begin{array}{l}\text { Outside } 95 \% \\
\text { of ND }(n=14)\end{array}$ & P-values \\
\hline Age (years) ${ }^{\$}$ & $67.1(13.3)$ & $61.7(13.7)$ & 0.7 & $68.6(12.7)$ & $64.2(14.5)$ & 0.6 \\
\hline $\begin{array}{l}\text { Body weight } \\
(\mathrm{kg})^{\$}\end{array}$ & $75(18.4)$ & $77.6(14.1)$ & 0.4 & 72.8 (19) & $81.6(12)$ & 0.2 \\
\hline $\operatorname{TTR}(\%)^{\$}$ & $65.6(17.2)$ & $64.7(16.4)$ & 0.6 & $65.7(16.1)$ & $65(21)$ & 1 \\
\hline $\mathrm{WSI}^{\$}$ & $0.7(0.4)$ & $0.3(0.1)$ & $0.0001^{*}$ & $0.8(0.4)$ & $0.3(0.1)$ & $0.0001^{*}$ \\
\hline $\begin{array}{l}\text { Log INR } \\
\text { variability }\end{array}$ & $-0.8(0.2)$ & $-0.9(0.2)$ & 0.06 & $-0.8(0.2)$ & $-0.9(0.2)$ & 0.06 \\
\hline $\mathrm{wCM}^{\$}$ & $-1.2(1.2)$ & $-1.8(0.9)$ & $0.004^{*}$ & $-1.1(1.2)$ & $-1.7(1.1)$ & 0.08 \\
\hline $\begin{array}{l}\text { Potentially } \\
\text { interacting } \\
\text { drugs } \\
\text { [n (\%)] }\end{array}$ & $123(61.2)$ & $12(63.2)$ & 0.9 & $135(65.5)$ & $8(57.1)$ & 0.5 \\
\hline
\end{tabular}



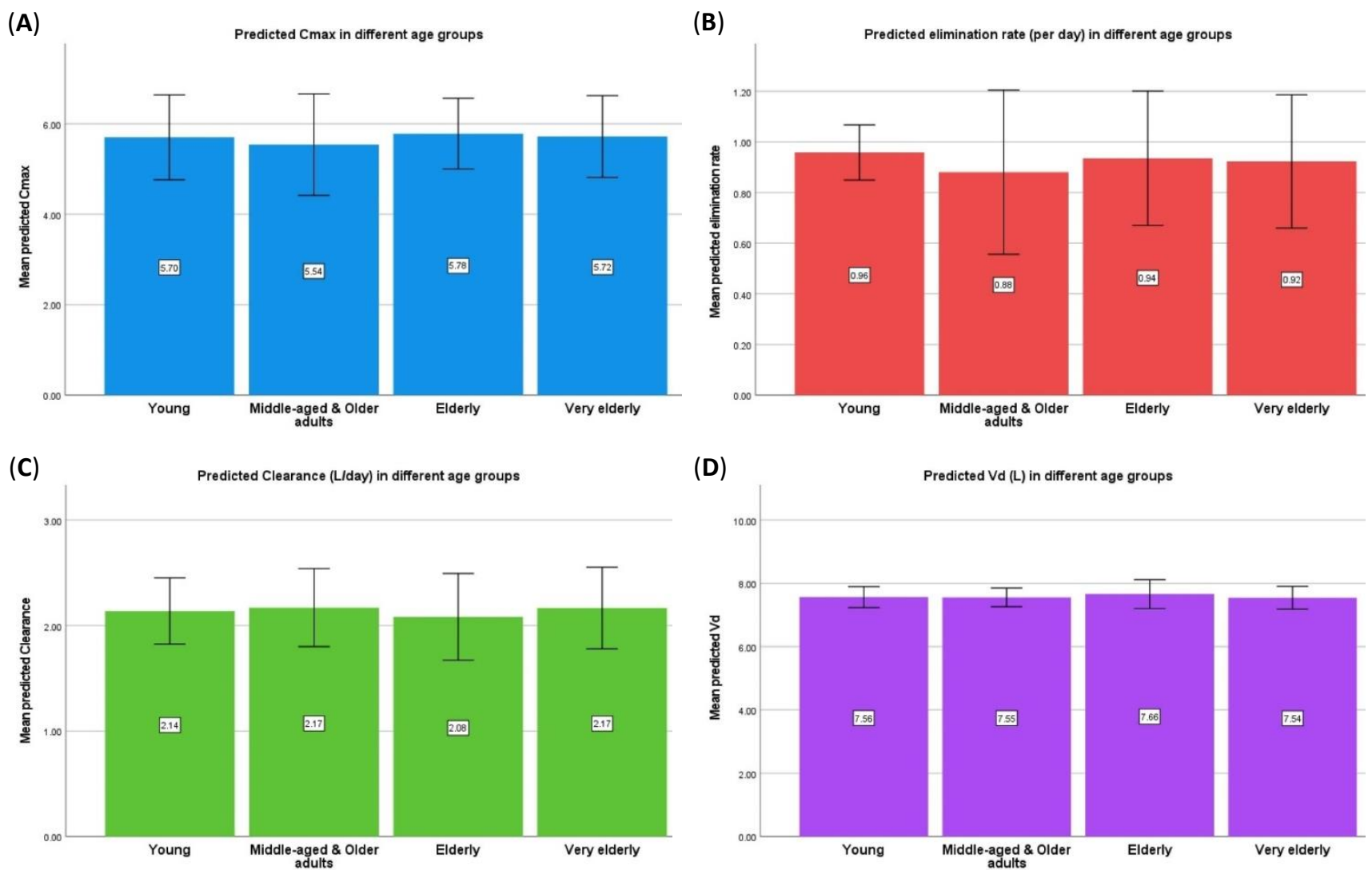

Figure 2. Predicted pharmacokinetic parameters of warfarin in different age groups. (A) Predicted $C_{\max }$ $(\mathrm{mg} / \mathrm{L})$; (B) predicted elimination (L/day); (C) predicted clearance (L/day); (D) predicted $V_{d}(\mathrm{~L})$ in different age groups.

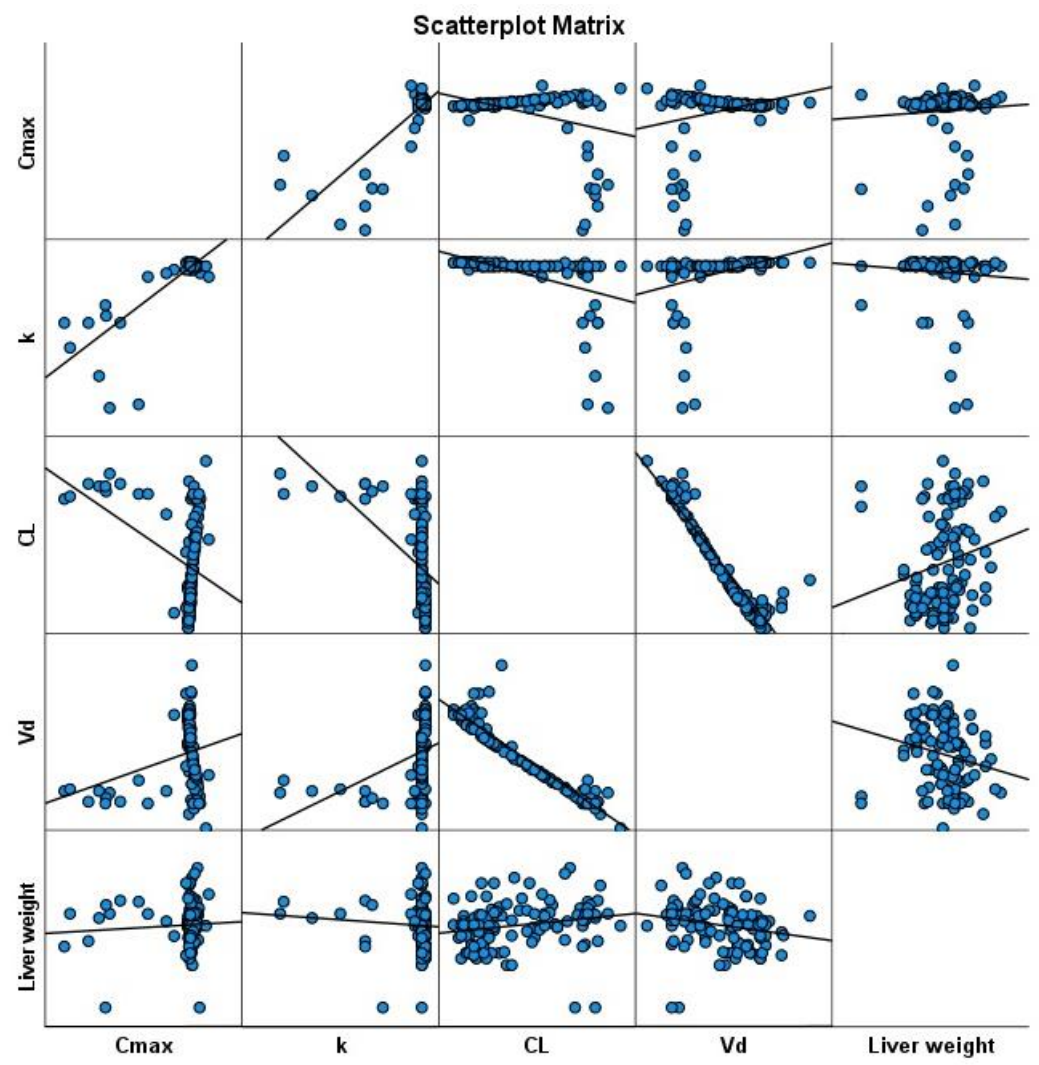

Figure 3. Scatter plot matrix between the pharmacokinetic parameters and the liver weight. Warfarin clearance and volume of distribution were significantly correlated with the weight of the liver. CL- clearance; $k$ - elimination rate; $V_{\mathrm{d}}$ - volume of distribution 


\section{Discussion}

We evaluated the pharmacokinetic parameters of warfarin using validated PK-PD model in our population. Two-hundred and twenty patients were recruited. The following were the predicted pharmacokinetic parameters: $C_{\max }(\mathrm{mg} / \mathrm{L})$ was 5.8 (0.4); elimination rate (L/day) was 1 (0.1); $\mathrm{CL}(\mathrm{L} /$ day) was 2.1 (0.2); and $V_{d}(L)$ was $7.6(0.2)$. Patients with $C_{\max }$ and elimination rate outside the mean \pm 1.96 SD had significantly lower WSI and higher WCM. Significant correlations were observed between $C_{\max }$ with $\mathrm{CL}, V_{\mathrm{d}}$, and $k$ of warfarin. Significant correlations were also observed between $C L$ and $V_{d}$ of warfarin with liver weight of the study participants.

The pharmacokinetic parameters of racemic warfarin observed in the present study is like other populations [14]. Although it would be better to measure $S$ - and $R$-warfarin concentrations, the clinical relevance of estimating enantiomers is limited. Ours is the first study exploring the relationship between pharmacokinetic parameters and WSI and WCM (the pharmacodynamic parameters). Lower WSI is corroborated with warfarin resistance. Genotyping of vitamin $K$ epoxide reductase complex in our population might provide a clue towards the prevalence and depth of warfarin resistance. The present study is also the first in exploring the relationship between liver weight and pharmacokinetic parameters of warfarin. Though, liver weight has been argued to be a better corroborator of pharmacokinetic parameters in children, [15] its relationship in adults has not been studied.

The study is limited in not validating the pharmacokinetic parameters using real-time measurements of plasma warfarin concentrations. To conclude, we predicted pharmacokinetic parameters of warfarin from the validated pharmacokinetic-pharmacodynamic model in our population. More studies are needed exploring the relationship between various pharmacodynamic indices of warfarin and pharmacokinetic parameters of warfarin.

Acknowledgements: We thank Research Technical Support Team, Ministry of Health, Kingdom of Bahrain for their Ethics approval of this study. We are grateful to AGU RCSI Steering Committee for their support in carrying out this research study.

\section{Conflict of interest: None}

Funding: The study was carried out as a part of the warfarin pharmacogenomics study that was funded by AGU RCSI Joint grant with grant number 2019-02.

\section{References}

[1] K. Harter, M. Levine, S.O. Henderson. Anticoagulation drug therapy: a review. West. J. Emerg. Med. 16(1) (2015) 11-17.

[2] C. Lv, C. Liu, Z. Yao et al. The clinical pharmacokinetics and pharmacodynamics of warfarin when combined with compound Danshen: A case study for combined treatment of coronary heart diseases with atrial fibrillation. Front Pharmacol. 8 (2017) 826.

[3] D.A. Barnette, B.P. Johnson, D.L. Pouncey et al. Stereospecific metabolism of R- and S-warfarin by human hepatic cytosolic reductases. Drug Metab. Dispos. 45(9) (2017) 1000-1007.

[4] J. Bubp, R. Coleman, D. Mungall, G. Raskob, R. Hull. Comparison of warfarin pharmacodynamic modelpredictions using Manchester comparative reagent vs Simplastin reagent. Clin. Pharmacol. Ther. 47 (1990) 157.

[5] D.A. Lane, G.Y. Lip. Use of the CHA(2)DS(2)-VASc and HAS-BLED scores to aid decision making for thromboprophylaxis in nonvalvular atrial fibrillation. Circulation 126 (2012) 860-865.

[6] G.Y. Lip. Atrial fibrillation and stroke prevention: brief observations on the last decade. Expert Rev. Cardiovasc. Ther. 12 (2014) 403-406. 
[7] C. de Fátima Colet, A.T. Amador, I. Heineck. Drug interactions and adverse events in a cohort of warfarin users attending public health clinics. International Journal of Cardiovascular Sciences 32 (2019) 110-117.

[8] Atrial fibrillation. Anticoagulation control. National Institute for Health and Care Excellence (NICE). Available at https://www.nice.org.uk/guidance/qs93/chapter/quality-statement-4-anticoagulationcontrol (Accessed on 15 July 2020).

[9] K. Sridharan, R. Al Banna, A.M. Qader, A Hussain. Evaluation of inter-patient variability in the pharmacodynamic indices of warfarin. Expert Rev. Cardiovasc. Ther. 18(11) (2020) 835-840. doi: https://doi.org/10.1080/14779072.2020.1814144.

[10] S.D. Fihn, A.A. Gadisseur, E. Pasterkamp, F.J. van der Meer, W.G. Breukink-Engbers, L.M. GevenBoere et al. Comparison of control and stability of oral anticoagulant therapy using acenocoumarol versus phenprocoumon. Thromb Haemost. 90(2) (2003) 260-266.

[11] Z. Razouki, J.F. Burgess Jr, A. Ozonoff, S. Zhao, D. Berlowitz, A.J. Rose. Improving anticoagulation measurement novel warfarin composite measure. Circ. Cardiovasc. Qual. Outcomes 8(6) (2015) 600607.

[12] S.C. Chan, C.L. Liu, C.M. Lo, B.K. Lam, E.W. Lee, Y. Wong, S.T. Fan. Estimating liver weight of adults by body weight and gender. World J. Gastroenterol. 12(14) (2006) 2217-2222.

[13] Java PK for Desktop software. Available at: http://pkpd.kmu.edu.tw/ipkd/index.php?pageNumrs news=1\&totalRows rs news=23 (Accessed on 21 Sep 20).

[14] A. Desai, T. Yamazaki, A.J. Dietz, D. Kowalski, C. Lademacher, H. Pearlman, S. Akhtar, R. Townsend. Pharmacokinetic and pharmacodynamic evaluation of the drug-drug interaction between isavuconazole and warfarin in healthy subjects. Clin. Pharmacol. Drug Dev. 6(1) (2017) 86-92.

[15] H. Takahashi, S. Ishikawa, S. Nomoto, Y. Nishigaki, F. Ando, T. Kashima, S. Kimura, M. Kanamori, H. Echizen. Developmental changes in pharmacokinetics and pharmacodynamics of warfarin enantiomers in Japanese children. Clin. Pharmacol. Ther. 68(5) (2000) 541-555. 